Research Article

\title{
Image Segmentation Method for an Illumination Highlight Region of Interior Design Effects Based on the Partial Differential Equation
}

\author{
Lina Wang, ${ }^{1}$ Yaoming Liu $\mathbb{D}^{2}{ }^{2}$ and Zhike Qian ${ }^{3}$ \\ ${ }^{1}$ Shanxi Vocational University of Engineering Science and Technology, Jinzhong 030600, China \\ ${ }^{2}$ Scientific Instrument Center, Shanxi University, Taiyuan 030006, China \\ ${ }^{3}$ College of Art and Design, Qingdao University of Technology, Qingdao 266000, China
}

Correspondence should be addressed to Yaoming Liu; liuym1022@sxu.edu.cn

Received 26 July 2021; Revised 9 August 2021; Accepted 16 August 2021; Published 28 August 2021

Academic Editor: Miaochao Chen

Copyright (c) 2021 Lina Wang et al. This is an open access article distributed under the Creative Commons Attribution License, which permits unrestricted use, distribution, and reproduction in any medium, provided the original work is properly cited.

\begin{abstract}
The saliency calculation model based on the principle of partial differential equations sometimes highlights areas with high contrast in the background, and the salient targets obtained occasionally have holes. The above problems can be solved by combining the improved convex hull calculation center saliency map. This paper designs a single-target color image segmentation algorithm based on partial differential equations. First, we calculate the basic saliency map according to the uniqueness of the color and the spatial distribution of the color; second, we then use the superpixel to improve the convex hull and calculate the central saliency map according to the principle; finally, the basic saliency map and the central saliency map are calculated. The weighted fusion is used to obtain the comprehensive saliency map, and the threshold method is used to segment the comprehensive saliency map to obtain the final target image. This paper designs an evaluation standard suitable for the segmentation of the illuminated highlight area of the effect image. It compares the experimental results of the segmentation method in this paper with the SLIC (Simple Linear Iterative Clustering) method and the traditional superpixel method to segment the illuminated highlight area. The segmentation method is applied to the image enhancement experiment. Based on the fuzzy means clustering algorithm, a fuzzy clustering objective function including brightness, color, and distance parameters is designed, which improves the weight of the brightness value in the clustering and improves the edge fit of the segmentation of the lighting highlight area of the rendering. The segmentation method produced by combining the clustering method with the superpixel biased clustering method can improve the output effect of the illuminated highlight area of the effect image after segmentation. We perform color equalization processing on the image to be segmented to reduce the impact of light, then set the closed value of the brightness information component, perform segmentation judgment, and expand the long and short axes of the ellipse model in the high-brightness area to further reduce the impact of light. The experimental results prove that the above method has a better segmentation effect than the traditional ellipse model and can accurately segment the gesture image. Compared with the existing mainstream saliency calculation models, this algorithm is closer to the true value image in terms of visual effects and has obvious advantages in terms of accuracy.
\end{abstract}

\section{Introduction}

The partial differential equation segmentation imaging system is a group of image imaging systems about the same target collected by multiple source channels. After image processing and computer technology, it can extract the beneficial information in each channel to the maximum extent and finally integrate it into a high-quality image [1]. Com- pared with the general digital imaging system, it has a higher spectral resolution, can easily and continuously change the wavelength of the output light, and coordinates hundreds of images of visible light and near-infrared light [2]. Therefore, partial differential equation segmentation imaging plays a very important role in color measurement, geospatial analysis, astronomy, and Raman chemical imaging [3]. However, because the partial differential equation 
segmentation imaging system has different reflectances of light in different bands to the same object, the brightness of different bands is very uneven, which results in the image information in the dark and highlight areas [4]. There is often a lot of detailed information with relatively small contrast in these areas. Once this information is lost, it will seriously affect the role of partial differential equation segmentation in its application field and actual production process. Therefore, it is of great significance to coordinate the definition of partial differential equations to segment digital images in these bands [5]. General image enhancement uses linear methods for filtering, such as convolution filtering, high-frequency enhancement filtering, and Wiener filtering. These methods are based on the actual application environment [6].

Images, videos, texts, and other content are all transmitted to the brain through human vision, and then, the brain extracts effective information to prepare for future image processing [7]. Machine vision is a system that uses machines to replace human eyes to identify image targets. It mainly includes three major steps: image segmentation, feature extraction, and target recognition [8]. Image segmentation is to divide the original image into several areas without intersection according to relevant rules. It is the premise and basis of image feature extraction and target recognition [9]. The result of this process will affect the success or failure of the subsequent operations to be processed, so when designing the color image segmentation method, the performance of all aspects of the algorithm should be improved as much as possible [10]. So far, color image segmentation algorithms are generally applied in specific scenes, and there is no general algorithm that can be applied to all types of image processing [11]. Most of the images collected by CCD cameras are in the form of RGB with a high correlation between brightness and chromaticity. Interior design effect images are very sensitive to changes in lighting, which makes it difficult to detect the pixels of interior design effect images, especially in high-brightness conditions [12].

In this paper, for the partial differential equation segmentation of the uneven-brightness image with large differences in different bands in the grayscale image, the enhancement method of improving the image contrast by improving the histogram equalization was performed, and an image enhancement method is cited based on the nonlinear partial differential equation to improve the clarity of partial differential equation segmentation of grayscale images. In order to objectively test whether the method is true and effective, while making the subjective judgment of the Human Visual System (HVS), it also introduces an objective function suitable for partial differential equation segmentation image definition evaluation-gray difference function, respectively. In order to improve the effective utilization of each band, this paper proposes an effective method to enhance the clarity of partial differential equation segmentation of grayscale images. Through nonlinear partial differential equations, the gradient space is expanded, the edges with larger gradient values are retained, and the texture details of the image are enhanced. Because the texture of the dark band of the partial differential equation segmentation image is weak, it is not easy to distinguish all its information. In view of the problems in the model, a new spread function is proposed, which can well preserve the edges and textures of the image while removing noise from the image, and the signal-to-noise ratio is significantly improved. In order to better fully reflect the enhancement effect, the histogram equalization is used to adjust the unevenness of the brightness. Finally, the clarity of this group of enhanced images is evaluated through two aspects: the qualitative aspect of human vision and the quantitative aspect of objective function. The results show that this method can effectively coordinate the definition of the partial differential equation segmentation image of each band, and the image enhancement effect is also very obvious.

\section{Related Works}

In the field of image processing, Malladi and Sethian [13] believe that the Markov Random Field (MRF) usually uses pixel-level features to model color images. Since MRF is equivalent to the Gibbs distribution, the joint distribution of MRF is changed to a simple model, using Bayesian maximum posterior probability estimation to calculate the cost function, so the color image segmentation task can be turned into a process of solving the cost function. Tagliabue et al. [14] proposed for the first time to optimize the cost function based on the idea of minimum cut and maximum flow. This process is called GrabCut, and this process was later applied in the field of image segmentation.

Dumitrescu et al. [15] designed a method based on the graph theory mentioned above, namely, GrabCut. GrabCut first transforms the image into a weighted undirected graph, where the sum of the weights of the edges in the undirected graph is the smallest. The edge is the correct dividing line we hope to obtain. According to the dividing line, the points in the undirected graph can be separated into two parts, namely, the background set and the target set of the image. GrabCut is a method based on energy optimization, but the algorithm also has certain flaws, that is, the NP-hard problem. Antonietti et al. [16] proposed GrabCut based on the problems of the GrabCut method, which is an algorithm that achieves the smallest energy function after multiple iterations. GrabCut uses a Gaussian mixture model to model the feature information of the foreground and background areas of the image. According to the color feature and edge feature of the original image and by adding a small number of manual interaction operations, it will obtain a high-accuracy effect map. Based on the research of the GrabCut algorithm, Malladi and Sethian [17] added a high-order potential energy term to record the feature information of a single pixel of the image and the adjacent information between pixels, which improved the accuracy of the Gaussian mixture model. At the same time, the Fully Convolution Network (FCN) algorithm is proposed. For all scales of color images to be processed, the end-to-end FCN framework is trained to complete the classification of all pixels in the image. This method can handle image semantic segmentation. The main design idea is that according to the zoom-out architecture, 
multilevel features can be obtained from images and applied to classification tasks.

In terms of image processing, researchers are more interested in visual saliency. The fundamental reason is that the visual attention mechanism can quickly find the area we hope to get from the massive amount of data. After Sheng et al. [18] first proposed the ITTI algorithm, scholars have successively constructed many visual saliency algorithms based on the ITTI algorithm. These algorithms are inspired by the ITTI model in their fundamental ideas. The ITTI algorithm is more suitable for the characteristics of HVS, but the design of the method is complicated and the algorithm runs slowly. In the ITTI model, the weights of the saliency maps generated by the three features are all the same, and the obtained saliency regions may be incomplete when applied to the image segmentation. We can select appropriate features according to specific application situations and adjust the weight of each feature in image processing [19]. In summary, the saliency map obtained according to the saliency calculation method of local contrast usually has a relatively high contrast value at the edge of the target, while the contrast value of the entire target of the image is relatively low, and the global contrast design is used. The model can uniformly highlight the salient target, but the edges of the target are not clear enough. Therefore, many scholars have tried to combine the two contrast methods, but when there is a high-contrast pixel set in the background of a color image, a model that only uses the contrast principle will misjudge the high-contrast area in the background as a salient target $[20,21]$.

\section{Construction of the Segmentation Model of the Interior Design Effect Image Based on Partial Differential Equations}

3.1. Distribution Space of Partial Differential Equations. In order to avoid equalizing the histogram while amplifying noise, this paper adopts an improved image enhancement method based on nonlinear partial differential equations and set the enhanced gradient image as $x(i, j)$ :

$$
x(i, j)=x(i+k, j+k), \quad i, j=1,2, \cdots, k
$$

Among them, $f(i)$ is the gradient function of the original image, and it is the maximum value of the gradient mode, which represents the direction information of the gradient field. After this transformation, the gradient function changes the original gradient field, and the distribution is transformed according to the required requirements, so that the original inconspicuous texture is highlighted, while retaining the edges with larger gradient values to enhance the texture details of the image.

$$
\frac{\partial f(i)}{\partial i}+\frac{\partial f(j)}{\partial j}=0
$$

After the image is enhanced, the image to be enhanced is restored through the principle of least squares. This process can enhance the weaker texture and the detailed information with a lower probability of occurrence in the image. In order to fully reflect the enhancement effect, this article uses the difference between the histogram equalization and the original image to multiply the compensation factor $u(x)$ to adjust the enhanced image, thereby constructing the reconstructed image; the formula is as follows:

$$
\begin{aligned}
& y(x)=[x(i) x(j)] *\left[\begin{array}{l}
x(i)^{\prime} \\
x(j)^{\prime}
\end{array}\right], \\
& u(x)=\sqrt{x(i) \cdot j^{2}+x(j) \cdot i^{2}} .
\end{aligned}
$$

Image sharpness is an important indicator to measure the effect of image enhancement. There are many objective function evaluations. The functions that reflect the detailed information of the image are entropy function, gray difference function, energy gradient function, and point sharpness function. Here, we choose the most suitable objective evaluation function for partial differential equation segmentation of digital image sharpness: gray difference function, to evaluate the original image and the enhanced image. Figure 1 shows a partial differential equation distribution spatial relationship diagram.

The algorithm of the gray difference function is as follows: first, we calculate the average gray value of each pixel one by one and then accumulate the difference between the gray value of each pixel and the average gray value to obtain the sharpness of the image. The formula is as follows:

$$
s(x)=\frac{e^{-x(i)}}{\lim _{n \rightarrow \infty} \sum_{i=1}^{n} \exp (x(i))} .
$$

There are many ways to eliminate the influence of light intensity on the image. First of all, the current image enhancement technology field is more mature, and it is also a basic method of image enhancement technologyhistogram equalization. It is a histogram correction method based on the cumulative distribution function (CDF). Its purpose is to correct the histogram of the original image into a balanced distribution form, that is, to equalize the gradient field of the original image and enhance the gradient field information with a high probability of appearing in the medium, suppressing the information with a low probability, so as to enhance the detailed information of the gray image in the dark band and the high-brightness band.

$$
\operatorname{Mean}(x)=\left|\frac{x(i)-x(j)}{x(i)+x(j)}+\frac{x(i)^{\prime}-x(j)^{\prime}}{x(i)^{\prime}+x(j)^{\prime}}\right| \cdot 100 \%
$$

For gray-scale image segmented by partial differential equation, the gray-level is divided. The main principle of the single Gaussian model is to establish a Gaussian model based on the probability distribution of the interior design effect image distribution. The parameters of this model are mean cents and covariance. According to the established 


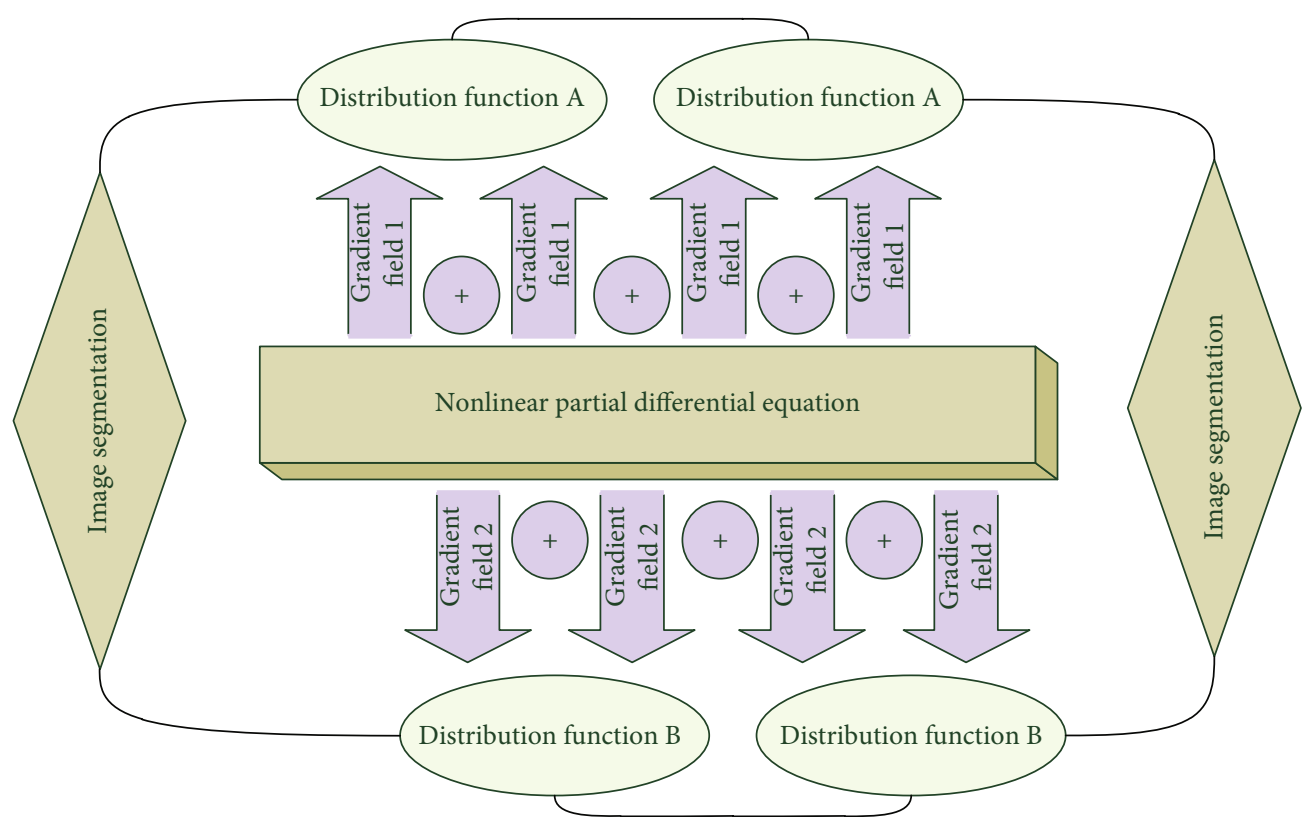

Figure 1: Partial differential equation distribution spatial relationship diagram.

model, we calculate the similarity between the measured pixel point $s(x, y)$ and the interior design effect image. The range of the $s$ value is $[0,1]$, and the similarity calculation formula is

$$
\operatorname{Div}(x, y)=s(x, y)-s(x-1, y)+s(x, y)-s(x, y-1) .
$$

In order to reduce the computational complexity, the Mahalanobis distance is used as a measure of the closeness between the input pixels and the image model of the interior design effect. Anisotropic diffusion not only diffuses in the vertical gradient direction but also diffuses in the gradient direction, and it is related to the gradient amplitude.

$$
\begin{gathered}
u(x, 0)=u_{0}(x) \cdot u(t, 0), \quad t>0, x \in R, \\
\left\{\begin{array}{l}
\frac{\partial g(x)}{\partial x}(x, t)-\Delta g(x, t)=0, \\
\left.\frac{\partial g(x)}{\partial x}\right|_{T}=0 .
\end{array}\right.
\end{gathered}
$$

When the image gradient is large, although the diffusion coefficient approaches zero, it can never be zero; that is, there is still diffusion between areas at the edges, which blurs the edges and textures. When the original image is heavily contaminated by noise or the contrast is very low, the resolution will greatly oscillate, resulting in unsatisfactory smoothing effects.

3.2. Image Lighting Highlight Algorithm. The threshold definition method is the most direct method for image segmentation of interior design effects. The main idea is to define a clear interval of skin color distribution. For example, in the $\mathrm{YCbCr}$ color space, through experiments and observations of a large number of interior design effect image samples, it is concluded that the interior design effect images on the plane $\mathrm{CbCr}$ are mainly concentrated in the range of $77<\mathrm{Cb}<127$ and $133<\mathrm{Cr}<173$.

$$
\frac{\partial u(x, y)}{\partial t}(t, x, y)+\frac{\partial x(t)}{\partial t}(t, x)=1
$$

For a pixel to be tested $u=(\mathrm{Cb}, \mathrm{Cr})$, this method classifies the pixel by judging whether the values of its two components fall in the interior design effect image interval.

$$
z=\lim _{n \longrightarrow \infty} \frac{1}{n} \sum_{i=1}^{N} u(x) \cdot x(i)-(t-x) \cdot(t-x)^{\prime} .
$$

The advantage of this method is that it can easily and quickly segment the gesture image, but the disadvantage is very obvious, its misjudgment rate is very high, and it is easy to confuse the noninterior design effect image area and the interior design effect image area. The single Gaussian model uses Gaussian distribution to fit the chromaticity probability density distribution of interior design effect images. Figure 2 shows the flowchart of the image light highlighting algorithm. Based on this principle, edge detection can be used to effectively segment the interior design effect image area and the noninterior design effect image area in the brightness subspace, and further, the area growth method is used to detect the normal brightness of the interior design effect image area to detect high-brightness interior design effect image pixels.

The single Gaussian model (SGM) method used has a significant interior design effect image clustering effect in the case of small samples, does not require a large number of interior design effect image samples, and does not require 


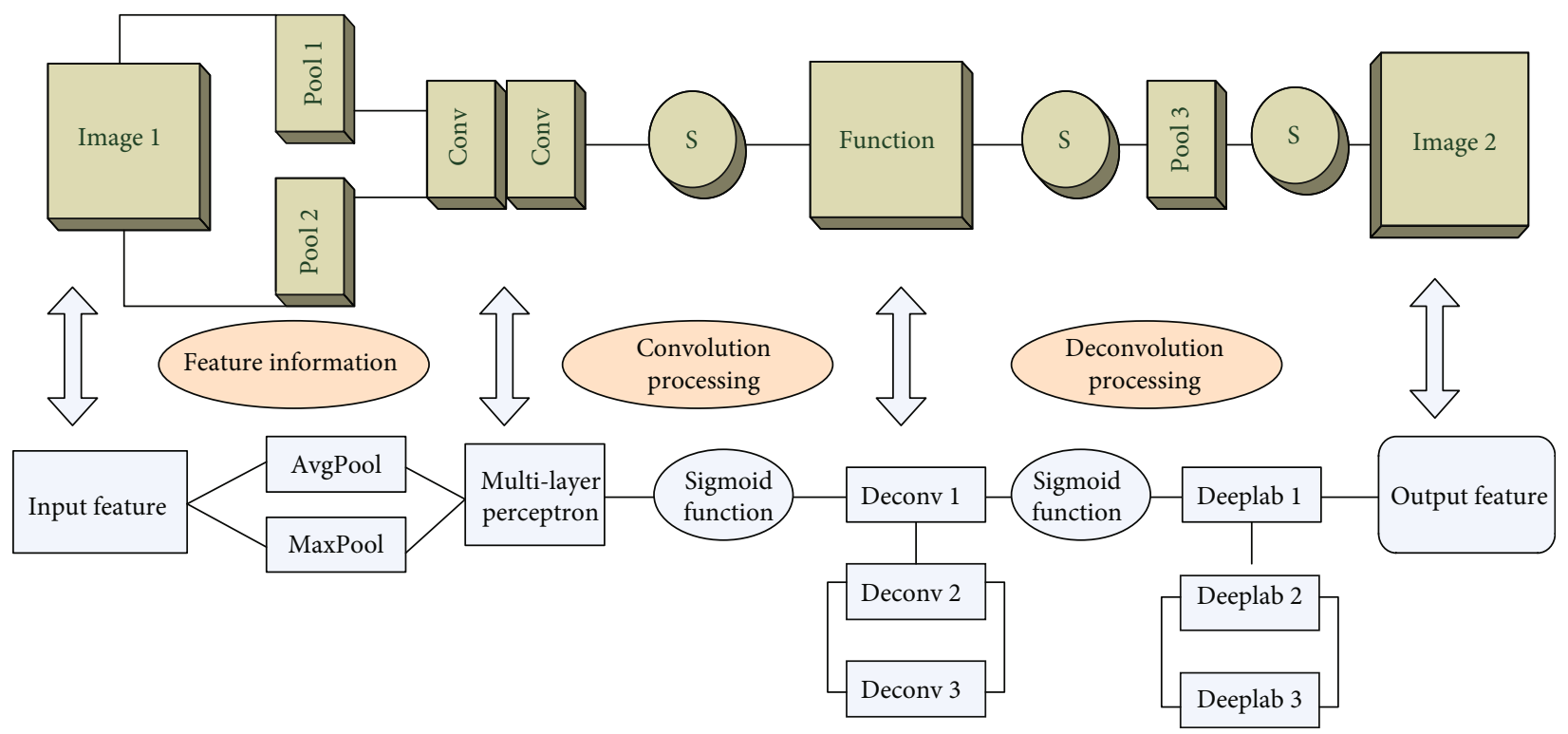

FIGURE 2: Flowchart of the image light highlighting algorithm.

a large amount of calculation during detection. The highbrightness interior design effect image pixel detection stage uses the human interior design effect image area brightness and spatial continuity principle to detect the highbrightness interior design effect image pixels based on the detection results of the previous stage.

$$
p(x \mid t)=\frac{1}{2 \pi \sqrt{s}} e^{-1 / 2(x-t)^{2} \cdot \sum 1 /(x-t)} .
$$

If the sample set is not processed uniformly, the model performance will be degraded due to the asymmetry of the sample space, which will affect the positive detection rate of the image points of the interior design effect. When the single Gaussian model is used to segment the interior design effect image, the segmentation result is not ideal when the light changes greatly. Before establishing an interior design effect image model, issues such as lighting changes should be considered. Therefore, it is necessary to perform color equalization processing on the segmented image. The highfrequency subband coefficients reflect the details and edge characteristics of the image and also include noise, which exists in the scale layer. Therefore, in this part of the image noise reduction processing, make the text more clear. Through the piecewise function, the necessary edge features are retained and interference is removed. When the brightness $Y$ changes, the distribution shape of the interior design effect image in the $\mathrm{CbCr}$ plane will be changed. Therefore, when the illumination changes, the color correction and light compensation of the image must be carried out. However, the light conditions of most images cannot be determined, so the gray world method must be used to perform color equalization on the segmented image.

3.3. Linear Optimization of Model Segmentation. After color equalization and improved ellipse model segmentation, a series of candidate regions are obtained, which can basically segment the gesture image from the complex background. However, in the background, there may be many areas that are similar to or the same as the human hand interior design effect image and are misjudged as human hand areas. It is impossible to completely eliminate these interferences. Therefore, it is necessary to further discriminate and optimize these candidate areas based on some prior knowledge and eliminate those areas that are obviously not manual. Through the previous experience and a large number of experiments, it has been found that considering the geometric features such as the shape and density of the image area of the interior design effect, a more satisfactory effect can be obtained. There is a nonlinear transformation relationship between the HSV color space and RGB color space.

$$
\left[\begin{array}{l}
\mathrm{Y} \\
\mathrm{K} \\
\mathrm{T}
\end{array}\right]=\left[\begin{array}{ccc}
u(0) & 0 & 0 \\
0 & \cdots & 0 \\
0 & 0 & u(t)
\end{array}\right] *\left[\begin{array}{l}
\mathrm{R} \\
\mathrm{G} \\
\mathrm{B}
\end{array}\right] .
$$

The Mahalanobis distance is calculated for each pixel, and a threshold is first set in the experiment to determine whether the current pixel is an interior design effect image pixel. If the Mahalanobis distance $C<1$, the point is judged to be an interior design effect image point; otherwise, it is a noninterior design effect image point.

$$
C(x, y, t)= \begin{cases}1, & \frac{(x-t)^{2}}{a^{2}}+\frac{(y-t)^{2}}{b^{2}}<1, \\ 0, & \text { others. }\end{cases}
$$

The value of the threshold value here directly affects the results of interior design effect image detection. If it is too large, the false detection rate of interior design effect image detection will be large; on the contrary, if it is too small, the false detection rate of interior design effect image 
detection will be large. In fact, the image content is different. It is obviously unreasonable to use a fixed threshold to segment the interior design effect image. It requires an adaptive interior design effect image segmentation algorithm.

Figure 3 shows the fan-shaped distribution diagram of the false detection rate of the interior design effect image detection after model segmentation. After the interior design effect image area under normal brightness has been detected, the human interior design effect image area brightness and spatial continuity principle proposed in the article can be used to detect the interior design effect image pixels in the high-brightness area. The interior design effect image detection algorithm based on the single Gaussian model is used to detect the interior design effect image in the normal brightness area of the image $f$, and the initial interior design effect image logo $r$ is obtained (the pixel point of the interior design effect image is set to 1 , and the rest of the pixel is set to 0 ). After grading, the original image is divided into three scale layers: coarse scale layer, detail scale layer, and fine scale layer. Coarse scale layer coefficients are lowfrequency coefficients, which contain the overview of the image. Fine scale layer coefficients are high-frequency coefficients, which reflect the details and edge characteristics of the image. Detail scale layer coefficients contain mediumand high-frequency coefficients, mainly including edge feature surface coefficients.

$$
\left[\begin{array}{l}
u \\
v
\end{array}\right]=\left[\begin{array}{cc}
\cos \theta & \sin \theta \\
-\sin \theta & \cos \theta
\end{array}\right] *\left[\begin{array}{cc}
C(a, b) & 0 \\
0 & C(b, a)
\end{array}\right] .
$$

For each brightness value $Y, 180>u>100$ and $160>v$ $>128$ are satisfied. The final interior design effect image logo map is output, and the interior design effect image area is extracted according to the interior design effect image logo map.

\section{Application and Analysis of the Segmentation Model Based on Partial Differential Equations of the Interior Design Effect Image Illumination Highlight Area}

4.1. Partial Differential Equation Segmentation Processing. This experiment was conducted on a computer configured with Intel Core i5-4200U $(1.6 \mathrm{GHz})$ with $4 \mathrm{G}$ memory and MATLAB 2011a programming experiment and used an external camera to collect gesture images. In order to show that the improved algorithm is superior to the histogram equalization algorithm, this paper selects the imaging system with higher imaging quality than the general partial differential equation segmentation imaging system, and under the D65 light source, the wavelength range collects a set of multispectral digital images for clarity analysis. Figure 4 shows the three-dimensional histogram of image quality in different illumination areas. The definition value of the original image, the image after the histogram equalization process, and the image processed by the improved method of the gray difference function are shown. This algorithm makes appropriate improvements to the traditional ellipse model.
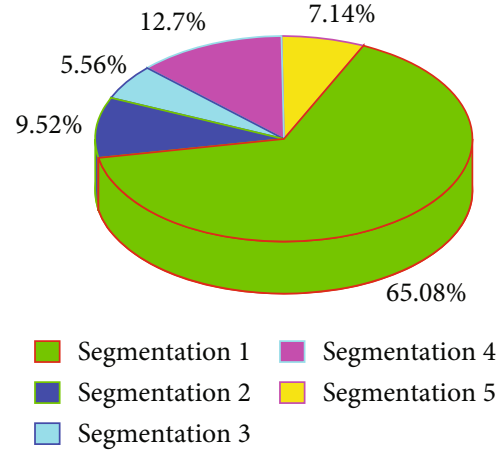

FIgURE 3: Fan-shaped distribution diagram of the false detection rate of interior design effect image detection after model segmentation.

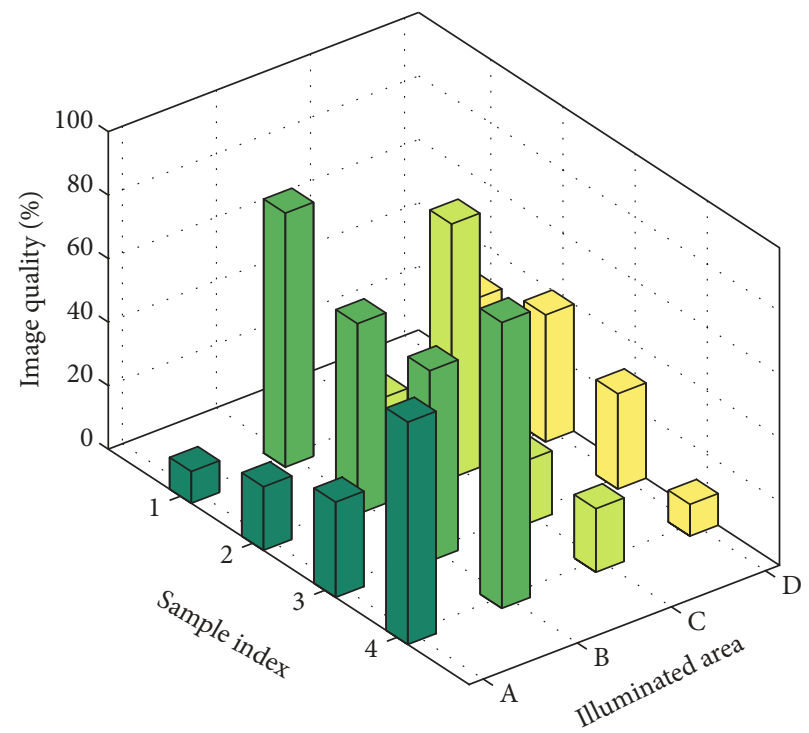

FIgURE 4: Three-dimensional histogram of image quality in different illumination areas.

When the brightness component $Y$ is greater than 230, we adjust the semimajor axis and semiminor axis of the ellipse model to 1.1 times of the original to further reduce the impact of illumination.

According to the shape characteristics of the local highbrightness area, it can be divided into band-shaped area and dot-shaped area. Figure 5 shows a schematic diagram of the partial differential equation linear division processing. In order to test the effectiveness of the method in this paper for processing high-brightness areas of different shapes in the text area and the blank area, two typical images are collected: the local high-brightness area is striped and distributed in the blank of the image area and text area. This method determines the thinnest path through the maximum value of the local gradient for values higher than a given threshold and uses these high gradient points for interpolation to construct a threshold surface to complete the binarization process. The band-shaped high-brightness area results in a variety of different brightnesses in the image; 


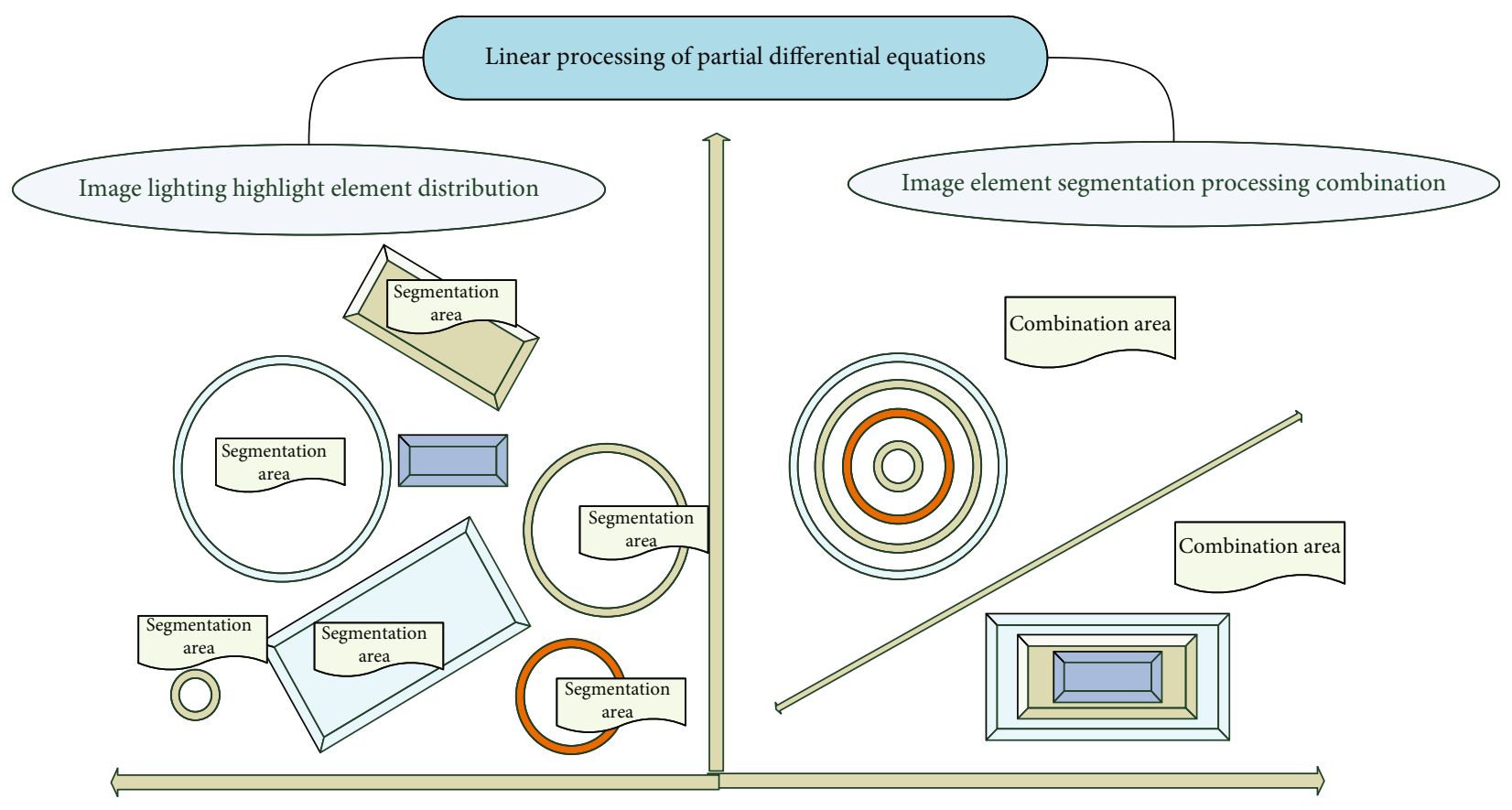

FIgURE 5: Schematic diagram of linear division processing of partial differential equations.

the local high-brightness areas are dotted and are also distributed in the blank and text areas.

Through observation, it is found that when the segmentation threshold changes from large to small, the number of pixels in the image judged to be interior design effect images will increase accordingly, and the rate of change in the number of pixels of interior design effect images at the optimal threshold is the smallest. Therefore, within the preset threshold range of 0.55 , the image is segmented in sequence with a step size of 0.05 and the change in the number of pixels belonging to the interior design effect image when the threshold changes is recorded. The optimal threshold is the threshold when the number of pixels of the effect image changes the least.

4.2. Interior Design Image Simulation. In order to verify the effect of the proposed high-brightness interior design effect image pixel detection algorithm based on the principle of human interior design effect image area brightness and spatial continuity, this paper tests multiple images with highbrightness interior design effect image pixel areas. The image size used in this experiment is 512 pixels $\times 512$ pixels, and the processing program is implemented in the environment of MATLAB 2011a. They are the processing results of bandshaped local high-brightness interfering text images and the processing results of dot-shaped local high-brightness interfering text images. Figure 6 shows the trend of the image noise value with the pixel band. It is not difficult to see that after the histogram equalization processing, the gray interval of the image histogram is enlarged, which is beneficial to the analysis and recognition of the image. However, this method has certain limitations. Although the sharpness of the 45band and 72-band images has been significantly improved, the image processed in the 55 bands and 60 bands will

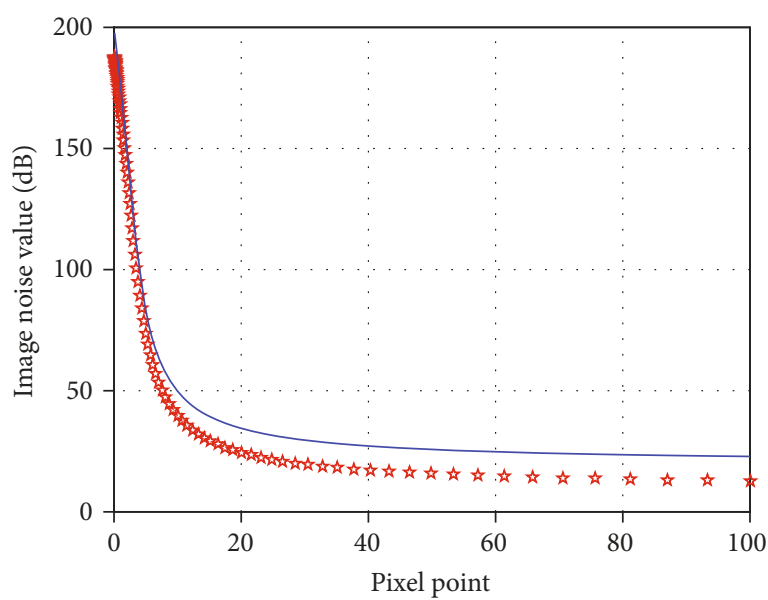

FIGURE 6: The trend of image noise value changes with pixel bands.

enlarge the image details with the histogram equalization. At the same time, the noise of the image is also amplified, especially in the 55 bands. It can be seen that this method not only has no obvious enhancement effect on the sharpness of 55-band grayscale images with relatively uniform illumination but also brings greater noise.

The parameters related to the experiment are set as follows: (1) Ncuts: in order to obtain a relatively high score, we set the number of blocks to 4. (2) Superpixel lattices: in order to ensure the consistency of the algorithm segmentation standard, the number of blocks is set to 100 . Figure 7 shows the histogram of the number of divisions in the highlight area of different images. When the number of superpixels is 80 , the number of superpixels is further reduced, and three umbrella-shaped areas are roughly distinguished; when the number of superpixels is 50 , the segmentation 
method in this paper will generate one in the most right umbrella-shaped illumination highlight area with the highest brightness. When the number is 20 , the right side 2 umbrella-shaped light highlight areas are divided, and when the number is 80 , the 3 umbrella-shaped light highlight areas are divided.

This method is also affected by the size of the window, and pen breaks and artifacts will occur when processing text images with uneven lighting and complex backgrounds. The method combines the edge and gray information of the image to generate a threshold surface and then uses the threshold surface to achieve binarization. The method first derives its gradient magnitude map according to the original image and then determines a gradient threshold. For values higher than a given threshold, the smallest path is determined by the local gradient maximum value, and these high gradient points are used for interpolation to construct a threshold surface. It can be seen from the above image characteristics that the visual effect of the binary image shown is relatively clear.

4.3. Example Application and Analysis. According to the requirements of the segmentation method in this paper, in order to verify the segmentation effect of the lighting highlight area of the effect image, it is necessary to include the effect image with extreme brightness areas in the test data set; that is, the images in the data set need to have some special high-brightness areas. This article selects 100 effect images with brightness differences, and the image resolution is between $320 \times 240$ and $2048 \times 1536$. The segmentation diagrams, respectively, show the cases where the number of superpixels is 5,20 , and 80 : when the number of superpixels is 5 , the method does not divide the elliptical area; when the number is 20 , the superpixels become smaller and the shape of the superpixels is honeycomb. When the number is 80 , the superpixels are further reduced, and the elliptical area is divided into dense honeycombs; when the number of superpixels is 50 , the segmentation method in this paper produces concentric elliptical superpixels in the elliptical illumination highlight area; and when the number is 20 , the segmentation distinguishes the chandelier and the surrounding roof lighting highlight area. Figure 8 shows the image resolution factor matchstick graph for different pixels. It can be seen that the interior design effect image pixel based on the single Gaussian model method cannot detect the high-brightness area in the image, and the highbrightness interior design effect image area detection algorithm proposed in the article can be more accurate based on the detection result of the single Gaussian model.

It can be seen that the elliptical and fan-shaped illuminated highlight areas have different degrees of overenhancement in the illuminated highlight areas. The visual effect highlighted by the enhancement is particularly obvious, which affects the effect of the image enhancement, which can also be seen from the experimental brightness distribution histogram. For the experimental results of the enhancement of the bright area of the effect image after segmentation, the image looks softer and uniform, and the enhancement effect displayed by the histogram of the bright-

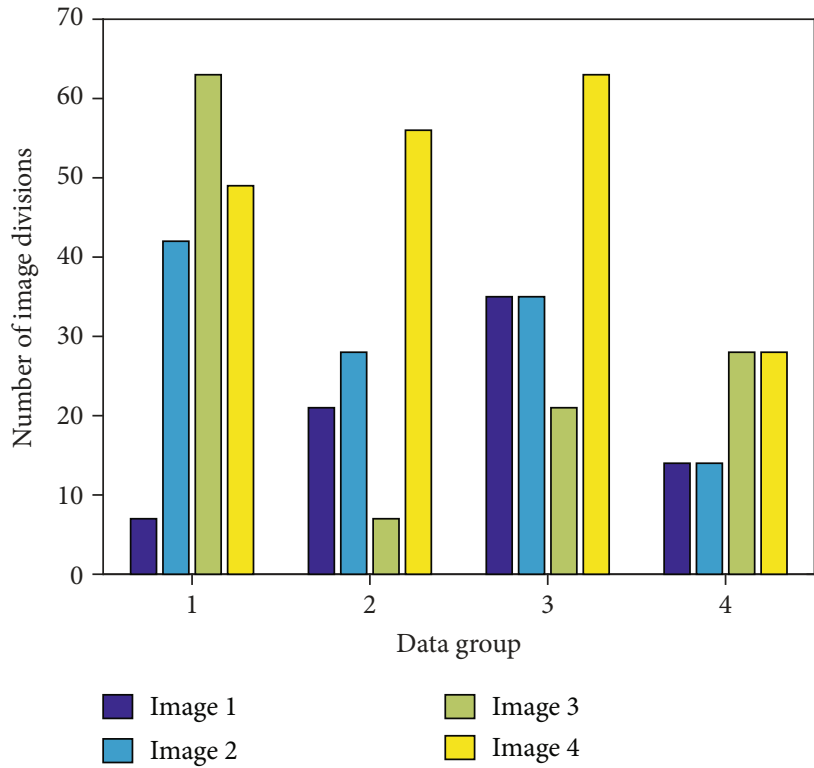

FIgURE 7: The histogram distribution of the number of divisions in the highlight area of different images.

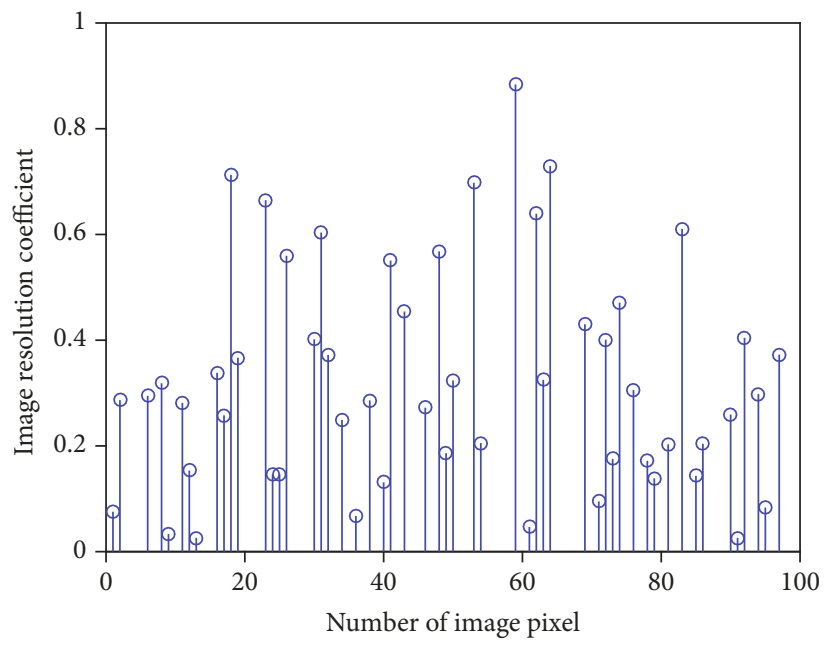

Figure 8: Image resolution coefficient matchstick diagram of different pixels.

ness distribution is natural and there is no excessive enhancement. Therefore, the method of segmentation of the illuminated highlight area of the effect image studied in this paper is applied to the effect image enhancement, which helps to improve the image enhancement effect. After being enhanced by the gradient field equalization enhancement method, the image is still very bright, and useful information cannot be expressed; after the algorithm in this paper is enhanced, the texture details on the switch that are originally unclear in the image can be highlighted, making the image very clear. By observing the experimental results, it can be seen that the method is not detailed enough and there are unclosed areas, because it takes the brightness factor into account. When the segmentation method in this paper is used to segment the bright area of the effect image, the single 


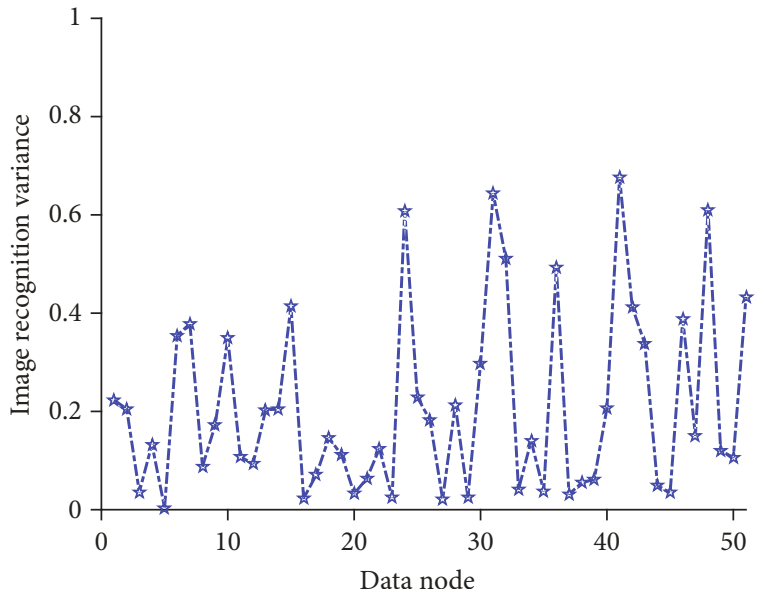

FIGURE 9: Line graph of recognition deviation after image segmentation.

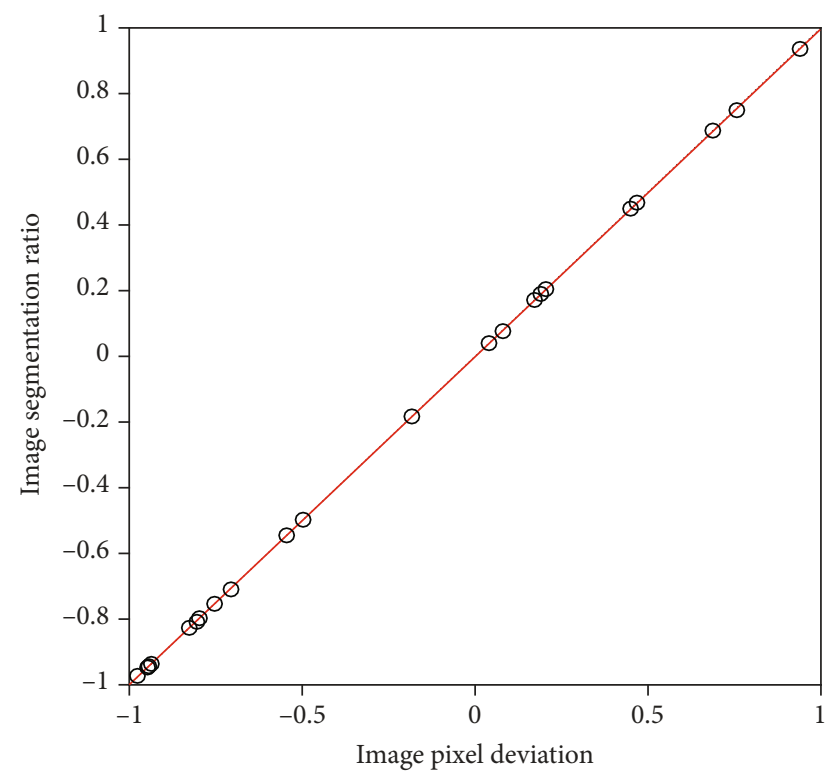

FIGURE 10: Linear fitting of the image superpixel segmentation ratio based on partial differential equations.

superpixel segmented is relatively large and the contour consistency rate is high.

After the two are superimposed, the pixel position and range of the misclassification and the omission can be clearly determined. Through the statistical calculation of the number of these wrong and missed pixels, the purpose of quantitatively analyzing the extraction accuracy can be achieved. Figure 9 shows the line graph of the recognition deviation after image segmentation. For different enhancement processing results of the same image, when the information entropy is relatively large, it means that the image enhancement processing makes the texture details in the original image appear more, the details are clearer, and the visual effect is better. In the test set, 50 frames of serial images were selected for the experiment, and the outline of the pore area in the first frame of serial images was manually marked. If multiple pore regions are to be processed, multiple extractions and then superimposition are needed to obtain the final result. The recognition degree of each group of images has increased from 0.55 to 0.61 and from 0.42 to 0.68 . From the data, it is obvious that the histogram equalization has limitations in some cases.

Figure 10 shows the linear fitting of the image superpixel segmentation ratio based on partial differential equations. By analyzing the generation of superpixels in the above situations, the following conclusions can be drawn: (1) as the number of superpixels increases, the superpixel segmentation effect produced by the two methods tends to be better. (2) When the number of superpixels is small, the super pixel segmentation boundary produced by the segmentation method in this paper is better. The result of the two segmentations has a great relationship with the shape of the light highlight area, the segmentation results obtained by the method have advantages, and the segmentation results obtained by the segmentation method in this paper are better for regions with long and narrow shapes and fuzzy edges. Therefore, in the case of a small number of superpixels, the segmentation results of the segmentation method in this paper are better.

\section{Conclusion}

In this paper, when using improved partial differential equations to calculate the central saliency of multitarget color images, some background saliency values will be high and the saliency value of the target will be low. In response to the above problems, we first use the boundary prior theory of partial differential equations to calculate the saliency map in the convex hull and weight the basic saliency maps to obtain a comprehensive saliency map; then, we use threshold segmentation to achieve image presegmentation. In order to improve the effect of interior design effect image segmentation, a method that can detect the high-brightness interior design effect image area in the image is proposed. The image pixels effectively solve the problem of the traditional interior design effect image detection method that misses the high-brightness interior design effect image area. This article first performs color equalization processing on the segmented image to reduce the impact of illumination, then sets the threshold for the brightness information component, performs segmentation judgment, and expands the long and short axes of the ellipse model in the highbrightness area to further reduce the impact of illumination. We realize the image detection of the interior design effect with the high-brightness interior design effect image area without increasing the sample size of the interior design effect image and storage space, which solves the problem that the detection of the interior design effect image using only the chromaticity information will affect the highbrightness interior. The experimental results prove that the method proposed in the article accurately detects the highbrightness interior design effect image area in the picture, while reducing the false detection rate, and can be applied to a wider range of brightness. 


\section{Data Availability}

The data used to support the findings of this study are available from the corresponding author upon request.

\section{Conflicts of Interest}

The authors declare that they have no known competing financial interests or personal relationships that could have appeared to influence the work reported in this paper.

\section{Acknowledgments}

This work was supported by the National Social Science Foundation of China: research on the supervision system of sports events in China (No. 19BTY018).

\section{References}

[1] D. Cheng, R. Liao, S. Fidler, and R. Urtasun, "DARNet: deep active ray network for building segmentation," in Computer Vision and Pattern Recognition, vol. 1, pp. 7431-7439, Long Beach, CA, USA, 2019.

[2] S. K. Weeratunga and C. Kamath, "Investigation of implicit active contours for scientific image segmentation," Society for Optics and Photonics, vol. 5308, pp. 210-221, 2020.

[3] Y. Achdou, F. J. Buera, J.-M. Lasry, P.-L. Lions, and B. Moll, "Partial differential equation models in macroeconomics," Philosophical Transactions of the Royal Society A: Mathematical, Physical and Engineering Sciences, vol. 372, no. 2028, pp. 30-37, 2018.

[4] J. Tumblin and G. Turk, "LCIS: a boundary hierarchy for detail-preserving contrast reduction," in Computer graphics and interactive techniques, vol. 2, pp. 83-90, USA, 2019.

[5] P. Houston and E. Süli, "A note on the design of hp-adaptive finite element methods for elliptic partial differential equations," Computer Methods in Applied Mechanics and Engineering, vol. 194, no. 2-5, pp. 229-243, 2020.

[6] J. Sirignano and K. Spiliopoulos, "DGM: a deep learning algorithm for solving partial differential equations," Journal of Computational Physics, vol. 375, pp. 1339-1364, 2018.

[7] C. Audouze, F. De Vuyst, and P. B. Nair, "Nonintrusive reduced-order modeling of parametrized time-dependent partial differential equations," Numerical Methods for Partial Differential Equations, vol. 29, no. 5, pp. 1587-1628, 2019.

[8] R. Raskar, K.-H. Tan, R. Feris, J. Yu, and M. Turk, "Nonphotorealistic camera: depth edge detection and stylized rendering using multi-flash imaging," in ACM transactions on graphics (TOG), vol. 23no. 3, pp. 679-688, New York, NY, USA, 2019.

[9] Y. Xu and C. W. Shu, "Local discontinuous Galerkin methods for high-order time-dependent partial differential equations," Communications in Computational Physics, vol. 7, no. 1, pp. 1-46, 2020.

[10] A. Pratt, L. Lari, O. Hovorka et al., "Enhanced oxidation of nanoparticles through strain-mediated ionic transport," Nature Materials, vol. 13, no. 1, pp. 26-30, 2018.

[11] F. Durand and J. Dorsey, "Fast bilateral filtering for the display of high-dynamic-range images," Computer Graphics and Interactive Techniques, vol. 2, pp. 257-266, 2020.
[12] S. Wang and M. Y. Wang, "Radial basis functions and level set method for structural topology optimization," International Journal for Numerical Methods in Engineering, vol. 65, no. 12, pp. 2060-2090, 2018.

[13] R. Malladi and J. A. Sethian, "Image processing: flows under min/max curvature and mean curvature," Graphical Models and Image Processing, vol. 58, no. 2, pp. 127-141, 2019.

[14] A. Tagliabue, L. Dede, and A. Quarteroni, "Isogeometric analysis and error estimates for high order partial differential equations in fluid dynamics," Computers \& Fluids, vol. 102, pp. 277-303, 2018.

[15] C. Dumitrescu, M. S. Raboaca, and R. A. Felseghi, "Methods for improving image quality for contour and textures analysis using new wavelet methods," Applied Sciences, vol. 11, no. 9, p. 3895, 2021.

[16] P. F. Antonietti, A. Cangiani, J. Collis et al., "Review of discontinuous Galerkin finite element methods for partial differential equations on complicated domains," Modern Approaches To Numerical Partial Differential Equations, vol. 6, pp. 281-310, 2019.

[17] R. Malladi and J. A. Sethian, "A unified approach to noise removal, image enhancement, and shape recovery," IEEE Transactions on Image Processing, vol. 5, no. 11, pp. 15541568, 2019.

[18] Y. Sheng, P. Willis, G. G. Castro, and H. Ugail, "Facial geometry parameterisation based on partial differential equations," Mathematical and Computer Modelling, vol. 54, no. 5-6, pp. 1536-1548, 2019.

[19] M. FRaissi, P. Perdikaris, and G. E. Karniadakis, "Inferring solutions of differential equations using noisy multi-fidelity data," Journal of Computational Physics, vol. 335, pp. 736746, 2017.

[20] C. Farhat, I. Harari, and U. Hetmaniuk, "The discontinuous enrichment method for multiscale analysis," Computer Methods in Applied Mechanics and Engineering, vol. 192, no. 28-30, pp. 3195-3209, 2019.

[21] J. O. Ramsay, "Differential equation models for statistical functions," Canadian Journal of Statistics, vol. 28, no. 2, pp. 225240, 2020. 